\section{A Rare Case of Myxoid Neurofibroma of the Penis and the Literature Review}

\author{
Keywords: Neurofibroma-1; nf-1 gene; penile Neurofibroma-1; \\ differential diagnosis

\section{Abstract} \\ Neurofibroma (NF) is an autosomal dominant genetic disease, which \\ can be divided into two subtypes: NF-1 and NF-2. In the genitourinary \\ system, NF-1 was usually found in the bladder, it in the penis is extremely \\ rare, to improve the diagnosis and treatment of the disease, in the present \\ case, myxoid NF-1 occurring in the penis was reported.
}

\section{Introduction}

Neurofibroma (NF) is an autosomal dominant genetic disease, which can be divided into two subtypes: NF-1 often occurs in peripheral nerves and is mainly related to mutations of $n f-1$ gene; NF-2 is rare, and it usually occurs in the central nervous system. NF-1 in the genitourinary system usually occurs in the bladder [1], however, NF-1 with myxoid degeneration occurring in the penis has not been reported. To improve the diagnosis and treatment of the disease, a 33-year-old patient with myxoid NF-1 of the penis has been reported in this case.

\section{Case Presentation}

A patient, a 33-year-old male, came to our center with the compliant for a penile mass for 1 month. He complained that a mass in penile was found accidentally, without sexual pain and abnormal erectile function. He had no trauma history in perineum. Since being noted, the mass has been progressively enlarged within the month. After admission, Physical examination revealed a healthy looking, not pale. No obvious abnormality was found in abdomen. Genitourinary examination showed that the patient had a mass in the dorsal center of the penis about $1.5 \mathrm{~cm}$ away from the coronal sulcus, with a size of about $0.8 \times 0.5 \mathrm{~cm}$ and no haphalgesia, no adhesion to the skin and spongy body, with good mobility. The foreskin is long, without edema. No swelling nodule was found in bilateral groin.

After admission, the patient has carried on the related preoperative examination. After excluding surgery contraindications, he was conducted $1 \%$ lidocaine local infiltration anesthesia for penile dorsal tumor resection, the mass in the dorsal center of the penis about $1.5 \mathrm{~cm}$ away from the coronal sulcus. While the mass was found closing to penile left dorsal nerve bundle (Figure 1), there is no adhesion and the surface is smooth (Figure 2). The surgical resection was carried out in a smooth manner, and the incision healed well one week after the operation. Postoperative pathological studies showed S100, CD34 and SOX10 were all positive deducing a diagnosis of myxoid NF-1 (Figure 3). The patients were followed up for 2 years after operation, and there was no recurrence, abnormal sexual function.

\section{Discussion}

$\mathrm{NF}$ is a common autosomal dominant genetic disease with a

\section{Journal of \\ Urology \& Nephrology}

\author{
ChengS ${ }^{1}$, Yang $\mathrm{J}^{2^{\star}}$, Zhang $\mathrm{Y}^{1}, \mathrm{Xu} \mathrm{An}{ }^{1}$, Dong $\mathrm{Li}^{1}$ and \\ Yang $\mathbf{G}^{1}$ \\ ${ }^{I}$ Department of Urology, Tongren Hospital, Shanghai Jiao Tong \\ University School of Medicine, China \\ ${ }^{2}$ Department of Pathology, Tongren Hospital, Shanghai Jiao Tong \\ University School of Medicine, China \\ *Address for Correspondence \\ Yang G, Department of Urology, Tongren Hospital, Shanghai Jiao \\ Tong University School of Medicine, Email: Yangg1103@126.com \\ Submission: 11 January, 2021 \\ Accepted: 15 February, 2021 \\ Published: 17 February, 2021 \\ Copyright: (๑) 2021 Cheng S, et al. This is an open access article \\ distributed under the Creative Commons Attribution License, which \\ permits unrestricted use, distribution, and reproduction in any medium, \\ provided the original work is properly cited.
}

prevalence of about $1 / 3000 \sim 1 / 3500$ [2]. NF can be classified into two subtypes according to the characteristics of skin lesions and neuropathy [3]: NF-1 often occurs in peripheral nerves and is mainly related to mutations of $n f-1$ gene, accounting for $90 \%$ of the total incidence. NF-1 is mostly superficial neurofibroma, the most common being plexiform neurofibroma. Plexus neurofibroma is considered to be a benign lesion histologically. The common components of neurofibroma are neuroaxons, Schwann cells, fibroblasts, mast cells, macrophages, peripheral nerve cells, and extracellular matrix, such as collagen. NF-1 is caused by the heterozygous mutation of $n f-1$ gene. The neurcellulose encoded by $n f-1$ gene has the function of expressing or down-regulating neurofibrin. When nf- 1 is mutated, it will lead to the loss of neurofibrin expression, thus leading to NF-1 lesions. $\mathrm{NF}-2$ is a subtype in which the central nervous system is frequently involved. The most common symptom is a sudden hearing loss, often caused by unilateral or bilateral vestibular schwannomas.

Pathologically, NF is a benign tumor of the nerve sheath, usually with soft or rubbery

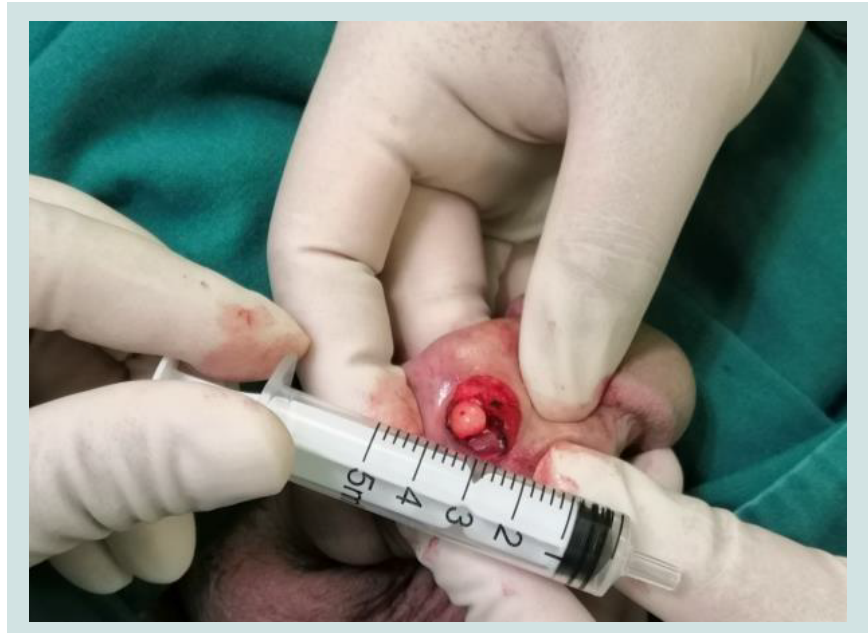

Figure 1: The mass locating in the dorsal of the penis. 
Citation: ChengS, Yang J, Zhang Y, Xu An, Dong Li, Yang G. A Rare Case of Myxoid Neurofibroma of the Penis and the Literature Review. J Urol Nephrol. $2021 ; 8(1): 2$

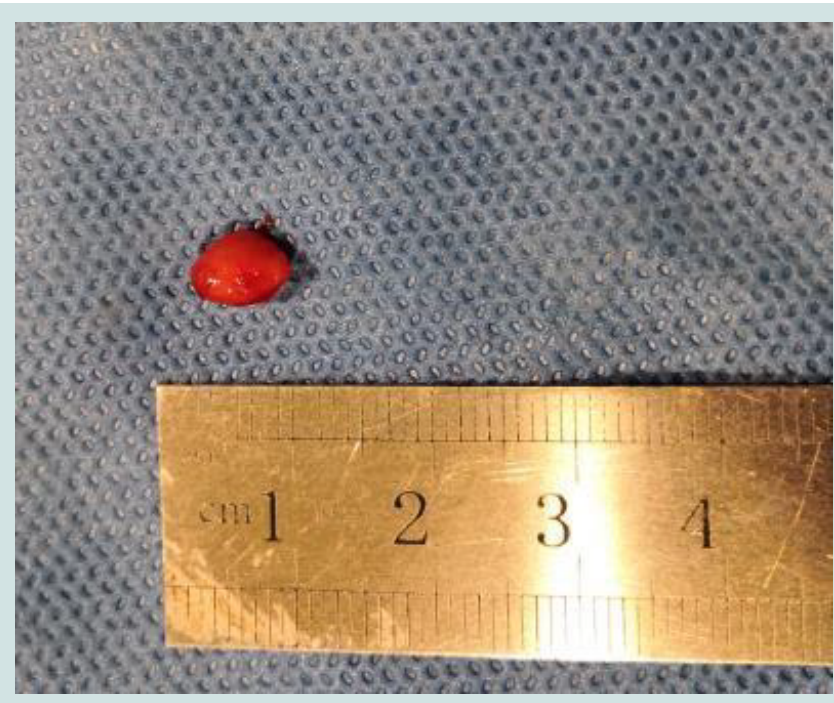

Figure 2: The gross appearance of the mass.

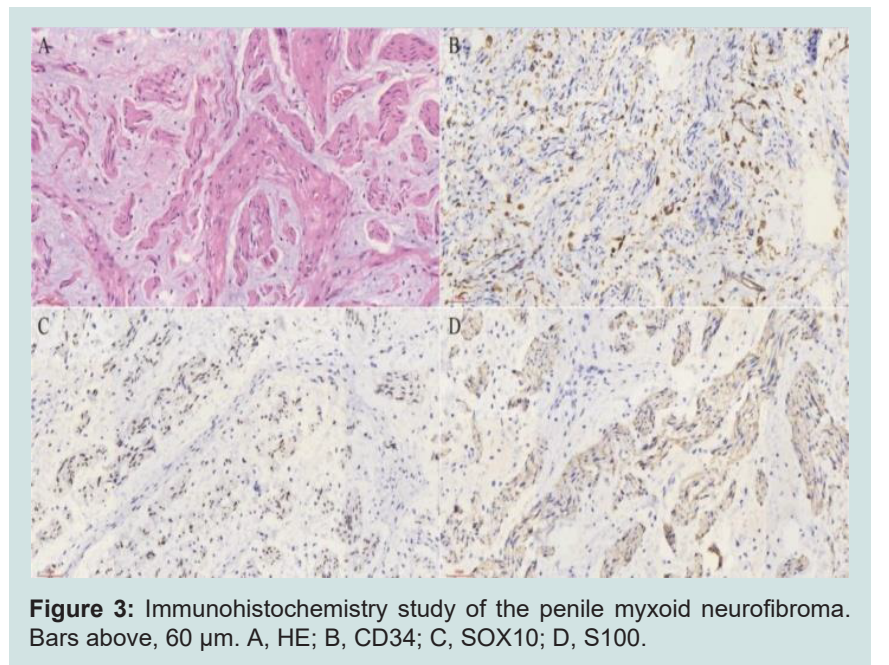

Pathologically, NF is a benign tumor of the nerve sheath, usually with soft or rubbery in texture [4]. Histopathological manifestations of NF include classical, myxiod, cellular, transparent, plexiform, epithelioid, diffuse, Parkini, pigmentation, and granulosa cell types. $\mathrm{NF}$ associated with mucin deposition including typical, mucinous, cellular, and plexiform nerve fibers [5].

NF-1 could occur in any part of the body, can continue to grow throughout life, and can be life-threatening due to compression of important structures, malignant transformation into neurosarcoma, or peripheral schwannomas. The lesions of NF-1 include milk and coffee spots on the skin, axillary or inguinal freckles, Lisch nodules on the iris, dysplasia of long bones and mental retardation. The lesions are mainly manifested as multiple nervous system tumors, skin pigmentation spots, vascular system and other visceral lesions. $\mathrm{NF}$ located in the penile body is reported in 1 case reported with the subtype of plexiform [3]. Although the myxoid NF often occurs in the face, shoulders and upper limbs, while myxoid NF in the penis has not been reported.

The differential diagnosis of NF-1 is commonly including tuberous sclerosis, McCune-abright syndrome and Proteus syndrome. The Myxoid differential diagnosis of NF should be distinguished from Spindle cell lipoma, Myxoma, Myxoid Liposarcoma, Myxoid Dermatofibromas Protuberans, and low-grade fibromyxoid Sarcoma. Although the occurrence of NF-1 in the penis is extremely rare, the possibility of the diagnosis should be considered to avoid misdiagnosis.

As a common benign lesion, the diagnosis of NF depends on the detection of pathological molecular markers, which could include S100 positive Schwann cells, CD34 and SOX10, and the 3 molecular markers in our case were all positive. The treatment of NF is mainly surgical resection, and the prognosis is good. The patient was regularly followed up for 2 years without tumor recurrence. There were several reports showed that the NFs in penis were benign lesions which have good consequence.

\section{Conclusion}

NF is an autosomal dominant hereditary disease. The Myxoid NF can also occur in the penis. Thus, when a lesion occurred in the site for diagnosis, Myxoid NF should be considered.

\section{References}

1. Ku S, Balasubramanian A, Kao CS, Eisenberg ML, Skinner EC (2020) Co-Manifestations of Genital Neurofibromatosis in a Patient with Neurofibromatosis Type 1 Urology 141: 49-50.

2. Brosius S (2010) A history of von Recklinghausen's NF1. J Hist Neurosci1 9: $333-348$.

3. Collins NC, Ayodeji EE, Motunrayo FO, Abayomi SB, Olufemi Ol, et al. ( 2018) Large penile plexiform neurofibroma in an 11-year old boy Malawi Med J 30: 49-51.

4. Erlandson RA, Woodruff JM (1982) Peripheral nerve sheath tumors: an electron microscopic study of 43 cases. Cancer 49: 273-287.

5. Megahed M (1994) Histopathological variants of neurofibroma. A study of 114 lesions. Am J Dermatopathol 16: 486-495.

\section{Acknowledgement}

This study was supported by the Med-Engineering Cross Foundation, Shanghai Jiaotong University (No. YG2019QNB28). 